(3.0-5.0 mm diameter) for chronic recording was drilled through the cranioplastic cap and the underlying skull.

\section{Behavioural task}

The rats were required to lick a spout to obtain rewards of $0.3 \mathrm{M}$ sucrose solution or ICSS (0.5-s train of $100-\mathrm{Hz}, 0.3-\mathrm{ms}$ capacitor-coupled negative square wave pulses). We monitored licking behaviour with a photoelectric sensor. The threshold level for ICSS to maintain the licking behaviour in the operant task was determined in behavioural tests before recording. In this study, the thresholds ranged from 65 to $110 \mu \mathrm{A}$. The licking frequency depended on the method of reward delivery, that is, low for ICSS and high for a liquid reward. This was probably because ICSS was delivered only once at the first lick even if the rat licked several times, whereas liquid was delivered continuously during a 2-s reward period, although the property of the reward, natural or artificial, could also be involved in this difference. In the cued operant task, a 1,200 Hz tone (tone 1) and a white light on the left side (light 1) signalled availability of the ICSS, and a $4,300 \mathrm{~Hz}$ tone (tone 2) signalled availability of sucrose solution. A $2,800 \mathrm{~Hz}$ tone (tone 3 ) and a white light on the right side (light 2) were neutral, that is, not associated with reward. A speaker positioned $10 \mathrm{~cm}$ anterior to the rats delivered tones that were $85 \mathrm{~dB}$ at each ear and two white light lamps, $45^{\circ}$ lateral and $7 \mathrm{~cm}$ from each eye, delivered the visual stimuli.

\section{Electrophysiology}

Before the unit recording session, we searched for auditory neural responses by clapping and, thus, localized the margins of the medial geniculate body. On the basis of the coordinates derived from this localizing procedure, a glass-insulated tungsten microelectrode $(Z=1.0-1.5 \mathrm{M} \Omega$ at $1,000 \mathrm{~Hz})$ was inserted stereotaxically stepwise by a micromanipulator into various parts of the posterior thalamus. Extracellular discharges of single neurons were recorded using conventional recording procedure.

\section{Histology}

At the end of recording session, several small electrolytic lesions $(20 \mu \mathrm{A}$ for $20 \mathrm{~s})$ wer made stereotaxically around the recording sites with a glass-insulated tungsten microelectrode. The brains were sectioned coronally $(30 \mu \mathrm{m})$ and stained with cresyl violet. Al marking and stimulation sites were then verified microscopically. The positions of the recorded neurons were mapped onto the appropriate tissue sections, using stereotaxi coordinates, and the sections were compared with the atlas of Paxinos and Watson ${ }^{29}$.

\section{Data analysis}

Spike trains were smoothed by convolution with a gaussian kernel ( $\sigma=20 \mathrm{~ms}$ ) to obtain spike-density functions (SDFs) ${ }^{30}$. Spontaneous activity was defined as the mean discharge rate during the $1,000 \mathrm{~ms}$ preceding cue onset. The early response was defined as the number of spike counts in the first $100 \mathrm{~ms}$ after cue onset. The late response was defined as the number of spike counts in the $500 \mathrm{~ms}$ period from 2,500 to $3,000 \mathrm{~ms}$ after cue onset. A neuron was classified as responsive to any cue stimuli if the difference in neura activity in three periods (spontaneous activity, early response and late response) was significant (one-way ANOVA, $P<0.01$ ). A modality coupling index $(\mathrm{MI})$ and a reward coupling index (RI) were computed for each response component: $\mathrm{MI}=\left(F_{\text {aud(ICSS) }}+\right.$ $\left.F_{\text {aud(ext) }}-F_{\text {vis }(\mathrm{ICSS})}-F_{\text {vis(ext })}\right) / F_{\text {sum }} ; \mathrm{RI}=\left(F_{\text {aud(ICSS })}-F_{\text {aud(ext })}+F_{\text {vis(ICSS })}-F_{\text {vis(ext })}\right) / F_{\text {sum }}$, where $F_{\text {sum }}=F_{\text {aud(ICSS) }}+F_{\text {aud(ext) }}+F_{\text {vis(ICSS) }}+F_{\text {vis(ext) }} \cdot F_{\text {aud(ICSS) }}$ or $F_{\text {vis(ICSS) }}$ is the average firing rate to auditory stimulus or visual stimulus, respectively, associated with reward minus spontaneous firing rate in each neuron. $F_{\text {aud(ext) }}$ or $F_{\text {vis(ext) }}$ is the average firing rate to auditory stimulus or visual stimulus, respectively, in the extinction test minus spontaneous firing rate in each neuron. MI represents sensory modality selectivity of the neura responses and RI represents reward dependency. For example, if the value of MI was 1.0 or -1.0 , this indicates a purely auditory-specific response or a visual one, respectively. An R value of 1.0 or 0 indicates that a neural response is totally dependent on or independent of reward contingency, respectively.

In principle, memory and anticipation are mutually dependent. However, the tempora response patterns (Fig. 1) and the effects of manipulating reward parameters (Figs 2 and 3 ) suggested that the early and late components conveyed distinguishable information.

Therefore we designated the early component as related to memory (retrospective coding) and the late one as related to anticipation (prospective coding).

\section{Received 26 January; accepted 13 June 2001}

1. Dickinson, A. \& Balleine, B. Motivational control of goal-directed action. Anim. Learn. Behav. 22, 1-18 (1994).

2. Schultz, W. Multiple reward signals in the brain. Nature Rev. Neurosci. 1, 199-207 (2000).

3. Schultz, W., Tremblay, L. \& Hollerman, J. R. Reward processing in primate orbitofrontal cortex and basal ganglia. Cereb. Cortex 10, 272-283 (2000).

4. Rolls, E. T. The Brain and Emotion. (Oxford Univ. Press, New York, 1999).

5. Rainer, G., Rao, S. C. \& Miller, E. K. Prospective coding for objects in primate prefrontal cortex. J. Neurosci. 19, 5493-5505 (1999)

6. Fibiger, H. C. \& Phillips, A. G. in Handbook of Physiology - The Nervous System Vol. IV (ed. Bloom, F. E.) 647-675 (Williams and Wilkins, Baltimore, 1986).

7. Wise, R. A. \& Rompre, P. P. Brain dopamine and reward. Annu. Rev. Psychol. 40, 191-225 (1989)

8. Mirenowicz, J. \& Schultz, W. Preferential activation of midbrain dopamine neurons by appetitive rather than aversive stimuli. Nature 379, 449-451 (1996).

9. Nakamura, K., Ono, T. \& Tamura, R. Central sites involved in the lateral hypothalamus conditioned neural responses to acoustic cues in the rat. J. Neurophysiol. 58, 1123-1148 (1987).

10. Robbins, T. W. \& Everitt, B. J. Neurobehavioral mechanism of reward and motivation. Curr. Opin. Neurobiol. 6, 228-236 (1996).
11. Ono, T., Nishijo, H. \& Uwano, T. Amygdala role in conditioned associative learning. Prog. Neurobiol. 46, 401-422 (1995).

12. Schoenbaum, G., Chiba, A. A. \& Gallagher, M. Orbitofrontal cortex and basolateral amygdala encode expected outcomes during learning. Nature Neurosci. 1, 155-159 (1998).

13. Bechara, A., Damasio, H., Tranel, D. \& Anderson, S. W. Dissociation of working memory from decision making within the human prefrontal cortex. J. Neurosci. 18, 428-437 (1998).

14. Watanabe, M. Reward expectancy in primate prefrontal neurons. Nature 382, 629-632 (1996).

15. Kawagoe, R., Takikawa, Y. \& Hikosaka, O. Expectation of reward modulates cognitive signals in the basal ganglia. Nature Neurosci. 1, 411-416 (1998).

16. LeDoux, J. E., Farb, C. R., \& Romanski, L. M. Overlapping projections to the amygdala and striatum from auditory processing areas of the thalamus and cortex. Neurosci. Lett. 134, 139-144 (1991).

17. Linke, R., De Lima, A. D., Schwegler, H. \& Pare, H.-C. Direct synaptic connections of axons from superior colliculus with identified thalamo-amygdaloid projection neurons in the rat: possible substrates of a subcortical visual pathway to the amygdala. J. Comp. Neurol. 403, 158-170 (1999).

18. Weinberger, N. M. Learning-induced changes of auditory receptive fields. Curr. Opin. Neurobiol. 3, 570-577 (1993)

19. LeDoux, J. E. Emotion circuits in the brain. Ann. Rev. Neurosci. 23, 155-184 (2000).

20. Dolan, R. J. in The New Cognitive Neurosciences 2nd edn (ed. Gazzaniga, M. S.) 1115-1131 (MIT Press, Cambridge, Massachusetts, 2000).

21. Gallistel, C. R., Leon, M., Waraczynski, M. \& Hanau, M. S. Effect of current on the maximum possible reward. Behav. Neurosci. 105, 901-912 (1991).

22. Bouton, M. E. Context, ambiguity, and classical conditioning. Curr. Direct. Psychol. Sci. 3, 49-53 (1994)

23. Bordi, F. \& LeDoux, J. E. Response properties of single units in areas of rat auditory thalamus that project to the amygdala. I. Acoustic discharge patterns and frequency receptive fields. Exp. Brain Res. 98, 261-274 (1994).

24. Edeline, J. M., Manunta, Y., Nodal, F. R. \& Bajo, V. M. Do auditory responses recorded from awake animals reflect the anatomical parcellation of the auditory thalamus? Hear. Res. 131, 135-152 (1999).

25. Winer, J. A. \& Morest, D. K. The medial division of the medial geniculate body of the cat: implications for thalamic organization. J. Neurosci. 3, 2629-2651 (1983).

26. Sefton, A. J. \& Dreher, B. in The Rat Nervous System 2nd edn (ed. Paxinos, G.) 833-898 (Academic, San Diego, 1995).

27. LaBerge, D. Thalamic and cortical mechanisms of attention suggested by recent positron emission tomographic experiments. J. Cogn. Neurosci. 2, 358-372 (1990).

28. Liu, Z., Murray, E. A. \& Richmond, B. J. Learning motivational significance of visual cues for reward schedules requires rhinal cortex. Nature Neurosci. 3, 1307-1315 (2000).

29. Paxinos, G. \& Watson, C. The Rat Brain in Stereotaxic Coordinates 4th edn (Academic, San Diego, 1998)

30. Richmond, B. J. \& Optican, L. M. Temporal encoding of two-dimensional patterns by single units in primate inferior temporal cortex. II. Quantification of response waveform. J. Neurophysiol. 57, $147-$ 161 (1987).

\section{Acknowledgements}

We thank R. Norgren (invited by Gofo Life Sciences International Fund) for helpful comments on this manuscript, and T. Kitamura for technical assistance. This work was partly supported by Grants-in-Aid for Scientific Research from the Japanese Ministry of Education, Science and Culture.

Correspondence and requests for materials should be addressed to T.O.

(e-mail: onotake@ms.toyama-mpu.ac.jp).

Practising orientation identification

improves orientation coding

\section{in V1 neurons}

\section{Aniek Schoups ${ }^{\star}$, Rufin Vogels ${ }^{\star}$, Ning Qian $† \&$ Guy Orban ${ }^{\star}$}

* Laboratorium voor Neuro-en Psychofysiologie, K.U. Leuven Medical School, B-3000 Leuven, Belgium

$\dagger$ Center for Neurobiology and Behavior, and Department of Physiology and Cellular Biophysics, Columbia University, New York 10032, USA

The adult brain shows remarkable plasticity, as demonstrated by the improvement in fine sensorial discriminations after intensive practice. The behavioural aspects of such perceptual learning are well documented, especially in the visual system ${ }^{1-8}$. Specificity for stimulus attributes clearly implicates an early cortical site, where receptive fields retain fine selectivity for these attributes; however, the neuronal correlates of a simple visual discrimination task remained unidentified. Here we report electrophysiological correlates in the primary visual cortex (V1) of monkeys for learning orientation identification. We link the behavioural improvement 
in this type of learning to an improved neuronal performance of trained compared to naive neurons. Improved long-term neuronal performance resulted from changes in the characteristics of orientation tuning of individual neurons. More particularly, the slope of the orientation tuning curve that was measured at the trained orientation increased only for the subgroup of trained neurons most likely to code the orientation identified by the monkey. No modifications of the tuning curve were observed for orientations for which the monkey had not been trained. Thus training induces a specific and efficient increase in neuronal sensitivity in V1.
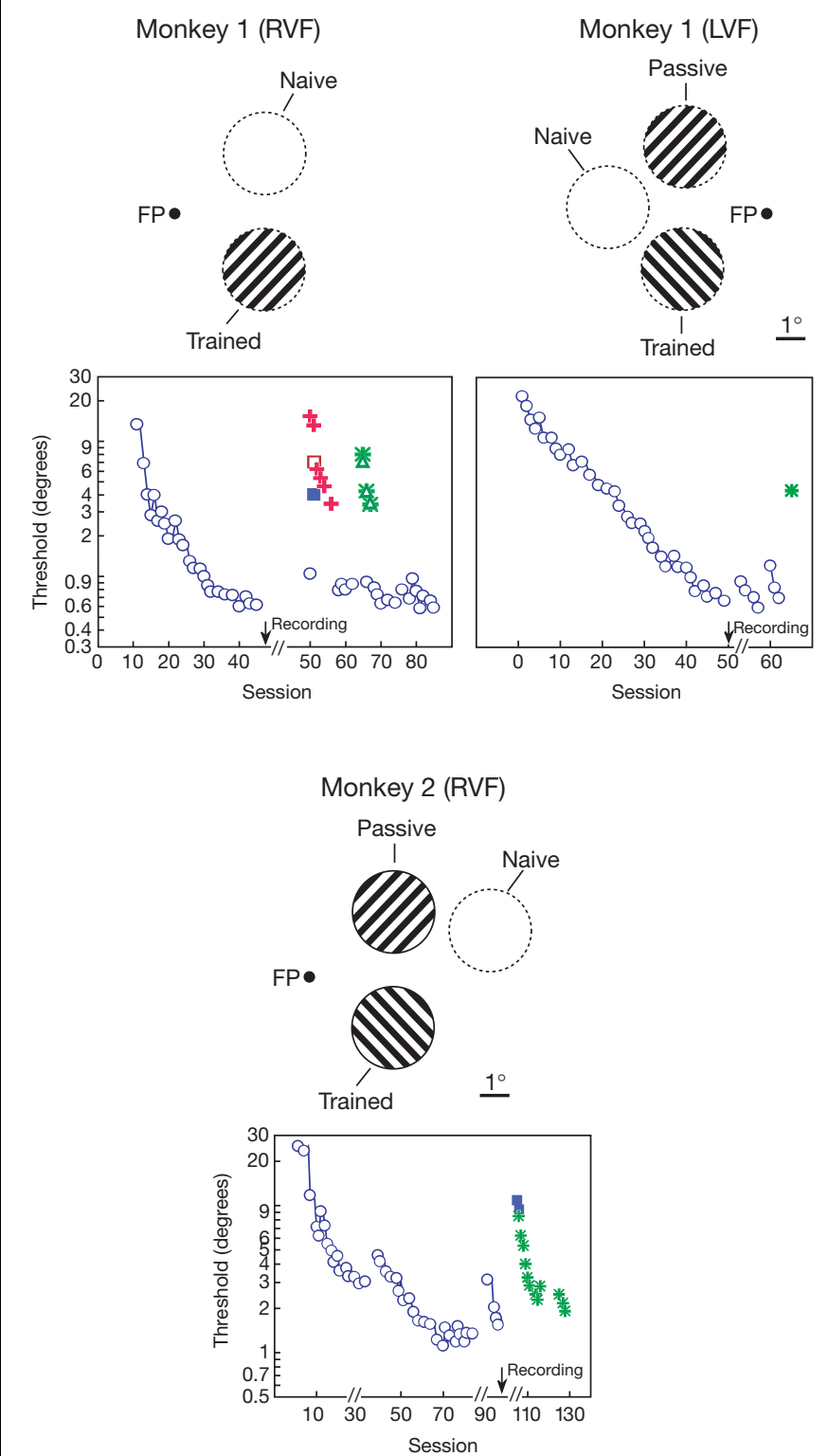

Figure 1 Behavioural performance and specificity for position and orientation. Learning curves for orientation identification for monkey 1 (two hemispheres) and monkey 2 (RVF). Above each learning curve, a schematic representation of the stimulus positions and reference orientation is given for each of the hemispheres studied. Thresholds for the trained orientation and position decreased on consecutive daily sessions (open blue circles). After recording, transfer was tested to the other oblique orientation (filled blue squares) at the same stimulus position, and to other stimulus positions (green, naive position; red, passively stimulated position). Thresholds at these other stimulus positions were similar for the two oblique orientations (green stars and triangles; red plus signs and open square), and were $7-12$ times larger than for the trained position and orientation. FP, fixation point.
The psychophysics of early perceptual learning has been well studied; however, researchers are only beginning to understand the neurophysiological correlates of perceptual learning. Primary somatosensory, motor and auditory cortex show learningdependent changes in their topographical organization ${ }^{9-11}$. In the visual system, however, early plasticity is demonstrated only by lesion-dependent reorganizations ${ }^{12,13}$. Improvement in motion discrimination, which transfers to other retinal stimulus positions, is accompanied by short-term improvements in neuronal sensitivity in the higher-order middle temporal and medial superior temporal areas within a single session, but does not extend across sessions ${ }^{14,15}$.

We trained two monkeys to identify the orientation of a small grating (Fig. 1). The performance of monkeys, like that of human subjects ${ }^{7}$, improved markedly with training, reaching a threshold as low as $0.6-1.2^{\circ}$ after several months. The improvement was specific for both stimulus position and orientation. This specificity provided us with an internal control: instead of comparing data between monkeys, we could compare different populations of

a

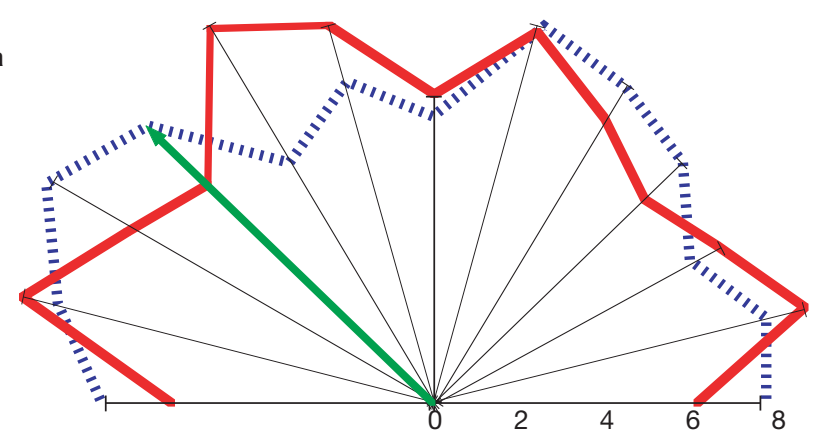

b
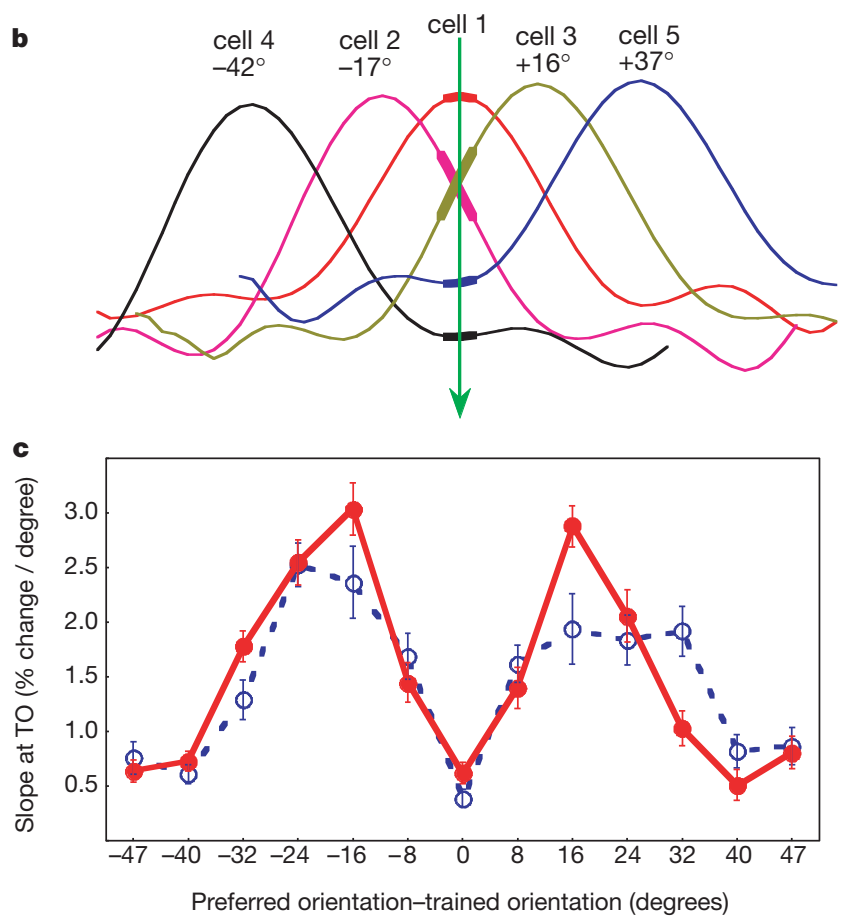

Figure 2 Neuronal responses. a, Distribution (\%) of preferred orientations in trained (solid red line) and naive neurons (dashed blue line). Data are from all cell layers. The green arrow indicates the trained orientation. $\mathbf{b}$, Orientation tuning curves of five sample neurons. c. Slope measured at trained orientation (TO) for trained (solid red line) and naive neurons (dashed blue line). Neurons are classified according to the angle between the preferred and trained orientation. Neurons tuned to the trained orientation are shown in the centre, groups with preferred orientation clockwise from trained orientation are to the left; those anticlockwise to the right. Data ( \pm s.e.m.) are from layers 2-3 and 5-6 combined. 
neurons within each hemisphere. The 'trained' population consisted of neurons with receptive fields at the trained position. A first control group (passive) consisted of cells responding to a second grating presented equally frequently during training, but that was behaviourally ignored by the subject. Second, neurons with receptive fields outside the trained or passive locations were considered naive. The monkeys' performance was verified after the recordings. The thresholds at which they could discriminate untrained orientations at the trained position, or the trained orientation at the two types of control positions, always exceeded the thresholds for the trained orientation at the trained position (Fig. 1).

After training, we recorded from 1,430 single units in V1 while the monkeys were performing a fixation task. The purpose was to reveal changes in the tuning characteristics of trained neurons that could be responsible for the observed improvement in sensory discrimination. Contrary to expectation ${ }^{9-11}$, we did not observe any shift in preferred orientations in favour of the trained orientation. The optima of neurons were evenly distributed over all orientations both before and after training (Fig. 2a).

It was only when we grouped all cells according to their preferred orientation that a specific change in the response characteristics of the trained neurons became apparent. Figure $2 \mathrm{~b}$ shows examples of

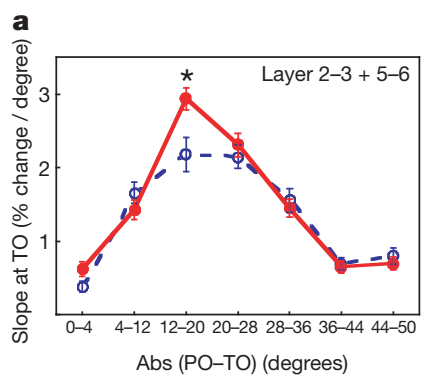

b

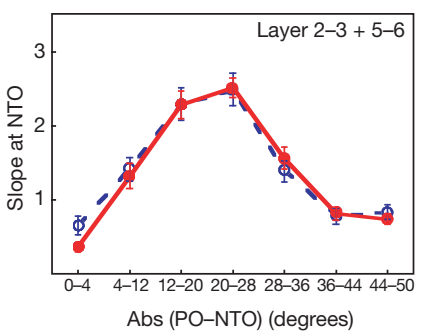

c

d

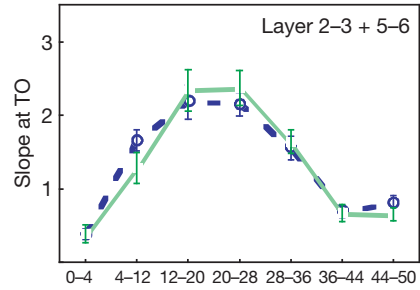

Abs (PO-TO) (degrees)

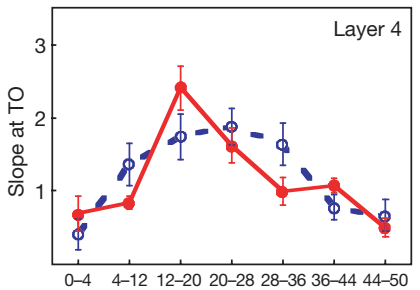

Abs (PO-TO) (degrees)

e

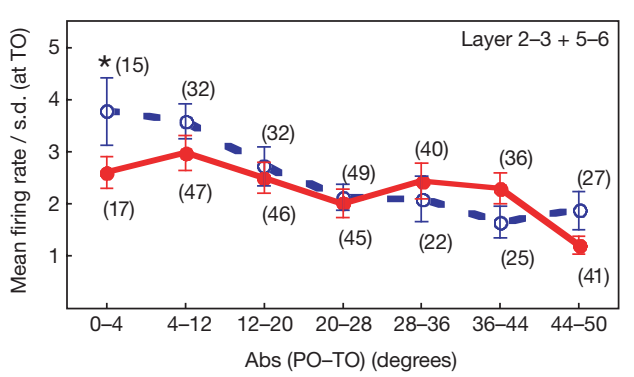

Figure 3 Neuronal responses. a, Increase in the slope of the tuning curve measured at the trained orientation (TO) for trained neurons tuned to orientations within 12 to $20^{\circ}$ of the trained orientation. $\mathbf{b}-\mathbf{d}$, This effect was not observed at the NTO (b), in passively stimulated neurons $(\mathbf{c})$, nor in trained neurons from layer $4(\mathbf{d})$. e, Decrease in the signalto-noise ratio for trained cells tuned to orientations within $0-4^{\circ}$ of the trained orientation. This ratio is given by the mean firing rate (averaged raw data) to the trained orientation, divided by the s.d. of the response distribution. The numbers of neurons for each preferred orientation (PO) group are indicated between brackets. In all panels trained neurons are indicated by a solid red line, naive neurons by dashed blue line, and passively stimulated neurons by dotted green line. *, significantly different. tuning curves, normalized to their respective maxima, with the preferred orientation indicated above each tuning curve. We measured the slopes of the tuning curves at the trained orientation (indicated by the thickening of the curves in Fig. 2b). The slopes are small, both for neurons that have their optima at or near the trained orientation (cell 1), and for those that do not respond to the trained orientation (for example cells 4 and 5). These neurons provide little information useful for detecting orientation differences. In contrast, the slope at the trained orientation is largest for neurons whose optimum differs by about $20^{\circ}$ from the trained orientation (for example cells 2 and 3). The firing rate of these neurons show the greatest change in response to a $1^{\circ}$ change in orientation, representing the highest sensitivity to small orientation differences ${ }^{16-18}$. Figure $2 \mathrm{c}$ plots the slope values as a function of angle (preferred orientation to trained orientation (PO-TO)) for cells with preferred orientations lying within $50^{\circ}$ of the trained orientation. At the point at which sensitivity is exactly most acute (that is, trained neurons with preferred orientations lying between 12 and $20^{\circ}$ of the trained orientation), an increase in the slopes of the tuning curves is observed. The increase in slope was consistent in that it was observed for trained cells with optima lying on either side of the trained orientation (Fig. 2c). In Figure 3a, both sides are combined, and the slope at the trained orientation is shown for the cells grouped according to the absolute value of the angle between the preferred orientation and the trained orientation. A significant main effect of angle (PO-TO) was observed, as well as a significant interaction between training and angle $(\mathrm{PO}-\mathrm{TO})\left(F_{6,492}=2.88\right.$; $P<0.009$ ). Post hoc analysis (least significant difference) showed that training has a significant effect only for the group of neurons with optima between 12 and $20^{\circ}$ of the trained orientation. This difference was observed in all three hemispheres studied (slopes for trained neurons with preferred orientations between 12 and $20^{\circ}$ of the trained orientation: $3.1 \pm 0.24,3.0 \pm 0.25,2.7 \pm 0.29$; for naive
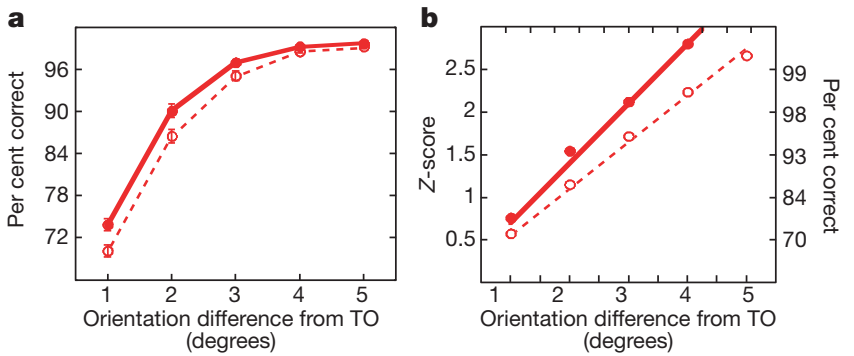

c

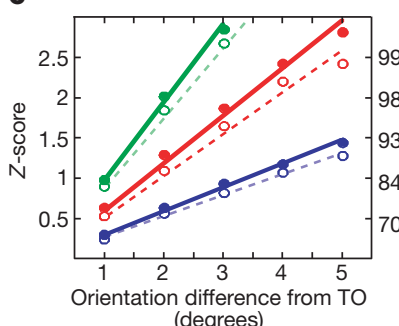

d

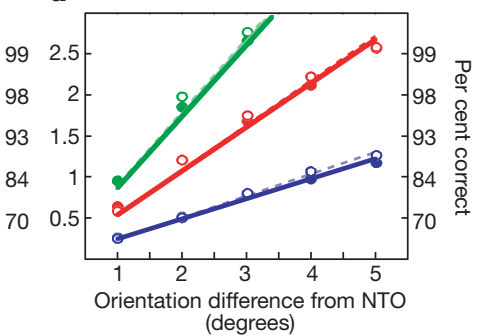

Figure 4 Neuronal performance. a, Percentage correct for a set of 20 cells, chosen from all trained or naive neurons tuned to orientations within $45^{\circ}$ of the trained orientation (TO), in identifying orientation differences around the trained orientation. $\mathbf{b}$, Performance for 20 cells randomly selected from the subset of neurons tuned to $12-20^{\circ}$ from the trained orientation. Slope is $27 \%$ higher for trained neurons compared with naive neurons.

c, $z$-transformed per cent correct as a function of orientation difference for various set sizes (20 cells: red, same data as in a; 5 cells: blue; 50 cells: green) randomly selected out of all trained or naive cells. Trained neurons always have a significantly steeper slope than naive neurons. Improvement for 5, 20 and 50 cells amounted to $12.1,12.5$ and $11.6 \%$, respectively. $\mathbf{d}$, Same as in $\mathbf{c}$, for identifying the untrained oblique orientation. Trained neurons have the same slope as naive neurones, for any set size of neurones selected. For all panels, solid lines indicate trained neurons; dashed lines indicate naive neurons. 
neurons: $2.2 \pm 0.34,2.3 \pm 0.39,1.9 \pm 0.59)$. As a first control, we performed the same analysis around the untrained oblique orientation (NTO). As described above, the monkeys had much larger thresholds for the NTO, $90^{\circ}$ from the trained orientation (Fig. 1, filled blue squares). Consistent with this behaviour, no significant differences were observed between naive and trained neurons for the slope measured at the NTO (Fig. $3 b ; F_{6,459}=0.42$; not significant). Figure $3 \mathrm{c}$ further shows that exposure to the stimulus alone was not sufficient to induce a change in the slope at the trained orientation, as passively stimulated cells failed to show this effect. Moreover, the changes in slopes of the tuning curve at the trained orientaion occurred in cells in the supra- and infragranular layers, but was not observed in those of input layer 4 (Fig. 3d).

The signal-to-noise ratio at the trained orientation is given by the mean firing rate in response to the trained orientation, divided by the s.d. A decrease in this ratio was observed for trained neurons tuned to orientations near the trained orientation (Fig. 3e; post hoc analysis $P<0.01$ ). If a monkey were to base its decision on the signals from the most active neurons, performance would deteriorate. The fact that it doesn't indicates once again that the difference signals arising from neurons whose relative activity changes the most sharply are the most relevant for the task ${ }^{16-18}$. The response variance, normalized to spike counts, remained unchanged after training, and averaged 1.85 and 1.90 spikes per $300 \mathrm{~ms}$ for naive and trained cells, respectively. No interaction between preferred orientation group and effect of training was observed $(P=0.88)$.

The increase in the slopes of the orientation tuning curves at the trained orientation was predicted by a recent computational model of orientation learning ${ }^{19}$ based on the recurrent models of orientation selectivity ${ }^{20,21}$. According to the model, reductions in neuronal activity at the trained orientation can lead to specific changes in the tuning curves, exactly as we have observed.

We next asked how relevant the observed changes in the orientation tuning curves to performance in the orientation discrimination task are. We calculated the neuronal discrimination abilities of all naive or trained cells using bayesian analysis ${ }^{22,23}$. Like any other reconstruction method it estimates the orientation presented from the observed neuronal activity. We used it to calculate the percentage of correct estimates for a given orientation difference. The orientation that has the highest probability of having occurred, given the neuronal response, was compared to the actual presented orientation for two orientations on either side of the trained orientation. The chief advantage of this method is that it performs like an ideal observer of population activity. Figure 4 a shows the neuronal discrimination performance of a set of 20 cells randomly selected from all naive or trained cells with preferred orientations within $45^{\circ}$ of the trained orientation. The neurometric curve for the trained population is shifted towards smaller orientation differences compared with the naive population. A cumulative normal distribution was fitted to these data points $(z \text {-transformation })^{7}$. After linear regression, only one parameter, the slope, defines the neuronal performance. The significantly greater slope (12\% increase; $P<$ $0.05, n=20$ ) for trained compared with naive cells in discriminating orientation differences around the trained orientation is shown in Fig. 4c. For increasing population sizes, the threshold decreases at a rate proportional to the square root of the number of cells. This hypothesis holds, provided that the firing of the neurons is not correlated, which was not taken into consideration when performing the bayesian analysis. The improvement in performance of trained versus naive cells, however, was independent of the set size (Fig. 4c). The observed change is quantitatively similar to the $13.6 \%$ improvement noted in motion-sensitive neurons of the middle temporal and medial superior temporal areas between two successive blocks of learning trials in a motion-discrimination task ${ }^{14}$. The difference between naive and trained neurons was not observed when orientation differences around the NTO were considered (Fig. 4d). Finally, when we consider only those neurons tuned to orientations between 12 and $20^{\circ}$ of the trained orientation, the neuronal improvement that accompanies training is much larger ( $27 \%$ increase in slope; Fig. $4 \mathrm{~b}$ ). Again, the trained group did not differ from naive cells for orientations of 1 to $5^{\circ}$ from the NTO (data not shown).

There are many reasons why the difference in neuronal performance between naive and trained cells did not equal the difference in thresholds for trained and untrained positions, observed psychophysically. On one hand, the behavioural improvement is probably overestimated. Improvement in a task like this one encompasses many behavioural aspects, and monkeys in particular are sensitive to any change in stimulus. Similar experiments with human subjects $^{7}$ revealed an improvement in psychophysical performance after learning of only $56 \%$ (increase in slope of the $z$-normalized psychometric curve). On the other hand, V1 may not be the sole locus of the sensorial improvement. Conversely, the neuronal performance could be underestimated. Although we used the most ideal classifier, it still does not take into account response correlations, which can improve discrimination and be affected by learning ${ }^{23-25}$.

A number of mechanisms have been suggested to explain various forms of perceptual learning. An expansion of the cortical representation has been demonstrated for tasks using features represented in topographical maps such as sound frequency or somatotopic localization $^{9-11}$. In our study, however, the proportion of cells that preferred the trained orientation remained unchanged. Neuronal noise reduction has also been suggested as an alternative mechanism $^{26}$. Our data, in accordance with that of others ${ }^{27}$, do not support this idea. Instead, we found that learning is correlated with specific changes in the tuning curves of specific groups of V1 cells. Only those cells that are relevant for signalling an orientation difference increase their discriminatory ability. Although our data do not preclude a multi-stage involvement, they show a first significant change in primary visual cortex.

\section{Methods \\ Psychophysics}

We trained two adult male rhesus monkeys (Macaca mulatta) in an orientation identification task. Monkey 1 was first trained to identify the right oblique reference orientation positioned in its right visual field (RVF), and after recording, was retrained using the left visual field (LVF) and the left oblique orientation. Monkey 2 was trained in the RVF, using the left oblique orientation. For two out of the three hemispheres, a second stimulus (passive) was placed in the upper visual field of the subject during training. The orientation of the second stimulus was randomly chosen from a narrow range of orientations $\left(0-5^{\circ}\right)$ around the oblique reference orientation, orthogonal to the one that had been trained. The monkey learned to ignore this stimulus. The monkeys had a stainless steel head-fixing device mounted to the skull and a scleral search coil implanted ${ }^{28}$ The stimulus was a sinusoidal grating, $2.6^{\circ}$ in diameter, 2.3 cycles per degree, contrast $90 \%$, positioned at $3.2^{\circ}$ eccentricity in the lower visual field. The phase was randomized so that orientation was the only cue that could be used to solve the task. A trial was initiated by the onset of a fixation point, which the subject had to fixate within a $1 \times 1^{\circ}$ window for $300 \mathrm{~ms}$. The subject had to hold fixation for another $250 \mathrm{~ms}$ while a grating was shown. Only one orientation, either tilted clockwise or anticlockwise with respect to the oblique reference orientation, was shown on each trial. After the stimulus was switched off, two response targets appeared above and below the stimulus position on which the subject had to make a saccadic eye movement to the correct target to receive apple juice. Once the subject had learned the task, training proceeded using a transformed up-down staircase procedure ${ }^{29}$, which converged on an orientation difference corresponding to an $84 \%$ correct criterion. Thresholds were defined as the logarithmic mean of the reversal points. The monkeys performed between 2,000 and 5,000 trials during each daily session.

\section{Electrophysiology}

We made extracellular recordings transdurally. The position of the receptive field was determined using multicellular activation by a small, flickering dot. When the response of a single cell was isolated, the optimal phase was determined and the response to 16

different orientations was recorded (15-30 trials per orientation). Spikes were counted in two 300-ms intervals, one before stimulus onset (baseline) and one starting $40 \mathrm{~ms}$ after stimulus onset (response).

We compared three populations of neurons: (1) trained neurons $(n=650)$; (2) passively stimulated neurons $(n=273)$; and (3) naive neurons $(n=507)$. Analysis of variance was applied to test for a significant response, and for an effect of orientation. From the 1,430 responsive single cells, 1,334 (93\%) were orientation tuned. Cells were assigned to supragranular, infragranular or granular layers on the basis of the spontaneous activity 
and depth of the electrode ${ }^{30}$. Cells from layers 2-3 and 5-6 combined comprised nearly $80 \%$ of the total (487 trained, 383 naive and 199 passively stimulated neurons).

Orientation tuning curves were normalized to their maximum and fitted using a polynomial of the 10th order. The squared sum of errors of the fitting averaged only 0.06 We omitted $7 \%$ (trained cells) to $10 \%$ (naive cells) of the cells because of a poor fit (squared sum of errors $>0.2$ ). We took the maximum of this fitted curve as the cell's preferred orientation and the tangent to the curve as the slope at the trained orientation, or at the untrained oblique orientation (NTO). Neurons were divided into 8-degree-wide groups according to the angle between their preferred orientation and trained orientation (or NTO). The numbers of neurons in each preferred orientation group are indicated between brackets in Fig. 3e. The slope changes derived from the fitted curves were independent of the fitting method, as similar results were obtained using a different order polynomial ( 8 or 12 ) or a spline fit.

Neurometric performance was determined using Bayes's rule ${ }^{22,23}$. Performance of an ideal classifier was measured by computing the probability that one of two orientations was presented, given the responses of a set of neurons. This set was randomly selected either from all cells with preferred orientations within $45^{\circ}$ of the trained orientation (naive $n=175$; trained $n=230$ ), from all cells with a preferred orientation within $45^{\circ}$ of the NTO (naive $n=170$; trained $n=224$ ), or from each preferred orientation group independently. Mean proportions of correct response are averages of 30 computations, each made on the basis of a new selection of cells. Each computation was based on 500 trials, taken from Poisson distribution derived from the mean firing rate of the cell. Mean responses to orientations, presented in $1^{\circ}$ steps, were taken from the normalized fitted polynomials and multiplied by the average maximum rate of that group of cells. Standard error of the mean of the per cent that were correct varied between $0.1-1.2 \%$. The values for per cent correct were $z$-transformed. After linear regression, the $84 \%$ threshold corresponds to the standard deviation $(z$-score of 1$)$.

Received 21 March; accepted 5 June 2001.

1. Ahissar, M. \& Hochstein, S. Task difficulty and the specificity of perceptual learning. Nature 387, $401-$ 406 (1997).

2. Crist, R. E., Kapadia, M. K., Westheimer, G. \& Gilbert, C. D. Perceptual learning of spatial localization: specificity for orientation, position, and context. J. Neurophysiol. 78, 2889-2894 (1997)

3. Fiorentini, A. \& Berardi, N. Perceptual learning specific for orientation and spatial frequency. Nature 287, 43-44 (1980).

4. Matthews, N., Liu, Z., Geesaman, B. J. \& Qian, N. Perceptual learning on orientation and direction discrimination. Vision Res. 39, 3692-3701 (1999).

5. Poggio, T., Fahle, M. \& Edelman, S. Fast perceptual learning in visual hyperacuity. Science 256, 1018 1021 (1992).

6. Vogels, R. \& Orban, G. A. The effect of practice on the oblique effect in line orientation judgements Vision Res. 25, 1679-1687 (1985).

7. Schoups, A. A., Vogels, R. \& Orban, G. A. Human perceptual learning in identifying the oblique orientation: retinotopy, orientation specificity and monocularity. J. Physiol. 483, 797-810 (1995).

8. Schoups, A. A. \& Orban, G. A. Interocular transfer in perceptual learning of a pop-out discrimination task. Proc. Natl Acad. Sci. USA 93, 7358-7362 (1996).

9. Recanzone, G. H., Merzenich, M. M., Jenkins, W. M., Grajski, K. A. \& Dinse, H. R. Topographic reorganization of the hand representation in cortical area $3 \mathrm{~b}$ of owl monkeys trained in a frequencydiscrimination task. J. Neurophysiol. 67, 1031-1056 (1992).

10. Recanzone, G. H., Schreiner, C. E. \& Merzenich, M. M. Plasticity in the frequency representation of primary auditory cortex following discrimination training in adult owl monkeys. J. Neurosci. 13, 87103 (1993).

11. Nudo, R. J., Milliken, G. W., Jenkins, W. M. \& Merzenich, M. M. Use-dependent alterations of movement representations in primary motor cortex of adult squirrel monkeys. J. Neurosci. 16, 785807 (1996).

12. Kaas, J. H. et al. Reorganization of retinotopic cortical maps in adult mammals after lesions of the retina. Science 248, 229-231 (1990).

13. Gilbert, C. D. \& Wiesel, T. N. Receptive field dynamics in adult primary visual cortex. Nature 356, 150-152 (1992).

14. Zohary, E., Celebrini, S., Britten, K. H. \& Newsome, W. T. Neuronal plasticity that underlies improvement in perceptual performance. Science 263, 1289-1292 (1994)

15. Zohary, E. \& Newsome, W. T. Perceptual learning in a direction discrimination task is not based upon enhanced neuronal sensitivity in the STS. Invest. Ophtalmol. Vis. Sci. 35, 1663 (1994).

16. Regan, D. \& Beverley, K. I. Postadaptation orientation discrimination. J. Opt. Soc. Am. A 2, 147-155 (1985)

17. Bradley, A., Skottun, B. C., Ohzawa, I., Sclar, G. \& Freeman, R. D. Visual orientation and spatial frequency discrimination: a comparison of single neurons and behavior. J. Neurophysiol. 57, 755-772 (1987).

18. Vogels, R. \& Orban, G. A. How well do response changes of striate neurons signal differences in orientation: a study in the discriminating monkey. J. Neurosci. 10, 3543-3558 (1990).

19. Qian, N. \& Matthews, N. A physiological theory for visual perceptual learning of orientation discrimination. Soc. Neurosci. Abs. 25, 1316 (1999).

20. Douglas, R. J., Koch, C., Mahowald, M., Martin, K. A. C. \& Suarez, H. H. Recurrent excitation in neocortical circuits. Science 269, 981-985 (1995).

21. Somers, D. C., Nelson, S. B. \& Sur, M. An emergent model of orientation selectivity in cat visual cortical simple cells. J. Neurosci. 15, 5448-5465 (1995).

22. Zhang, K., Ginzburg, I., McNaughton, B. \& Sejnowski, T. J. Interpreting neuronal population activity by reconstruction: unified framework with application to hippocampal place cells. J. Neurophysiol. 79, 1017-1044 (1998)

23. Oram, M. W., Foldiak, P., Perrett, D. I. \& Sengpiel, F. The 'ideal homunculus': decoding neural population signals. Trends Neurosci. 21, 259-265 (1998).

24. Panzeri, S., Schultz, S. R., Treves, A. \& Rolls, E. T. Correlations and the encoding of information in the nervous system. Proc. R. Soc. Lond. B 266, 1001-1012 (1999).

25. Recanzone, G. H., Merzenich, M. M. \& Schreiner, C. E. Changes in the distributed temporal response properties of SI cortical neurons reflect improvements in performance on a temporally based tactile discrimination task. J. Neurophysiol. 67, 1071-1091 (1992).
26. Dosher, B. A. \& Lu, Z. Perceptual learning reflects external noise filtering and internal noise reduction through channel reweighting. Proc. Natl Acad. Sci. USA 95, 13988-13993 (1998).

27. Gold, J., Bennett, P. J. \& Sekuler, A. B. Signal but not noise changes with perceptual learning. Nature 402, 176-178 (1999).

28. Judge, S. J., Richmond, B. J. \& Chu, F. C. Implantation of magnetic search coils for measurement of eye position: an improved method. Vision Res. 20, 535-538 (1980).

29. Wetherill, G. B. \& Levitt, H. Sequential estimation of points on a psychometric function. Brit. J. Math. Stat. Psychol. 18, 1-10 (1965).

30. Snodderly, D. M. \& Gur, M. Organization of striate cortex of alert, trained monkeys (Macaca fascicularis): ongoing activity, stimulus selectivity, and widths of receptice field activating regions J. Neurophysiol. 74, 2100-2125 (1995).

\section{Acknowledgements}

We would like to thank K. Claeys, P. Janssen, Z. Li, H. Op de Beeck, H. Peuskens, S. Raiguel, N. Sachs and W. Vanduffel for critical discussions, and M. DePaep, P. Kayenbergh, G. Meulemans, G. Vanparrijs for technical assistance. A.S. is supported by a fellowship from FWO. This project was funded by grants from FWO (A.S.) GSKE (R.V.) NSF and NIH (N.Q.) and from DWTC (G.O.)

Correspondence and requests for materials should be addressed to A.S (e-mail: annick.schoups@med.kuleuven.ac.be).

\section{Regulation of DNA replication fork progression through damaged DNA by the Mec1/Rad53 checkpoint}

\author{
José Antonio Tercero \& John F. X. Diffley
}

Imperial Cancer Research Fund, Clare Hall Laboratories, South Mimms EN6 3LD, UK

The checkpoint kinase proteins $\mathrm{Mecl}$ and Rad53 are required in the budding yeast, Saccharomyces cerevisiae, to maintain cell viability in the presence of drugs causing damage to DNA or arrest of DNA replication forks ${ }^{1-3}$. It is thought that they act by inhibiting cell cycle progression, allowing time for DNA repair to take place. Mec1 and Rad53 also slow S phase progression in response to DNA alkylation, although the mechanism for this and its relative importance in protecting cells from DNA damage have not been determined. Here we show that the DNA-alkylating agent methyl methanesulphonate (MMS) profoundly reduces the rate of DNA replication fork progression; however, this moderation does not require Rad53 or Mec1. The accelerated $S$ phase in checkpoint mutants ${ }^{4}$, therefore, is primarily a consequence of inappropriate initiation events ${ }^{5-7}$. Wild-type cells ultimately complete DNA replication in the presence of MMS. In contrast, replication forks in checkpoint mutants collapse irreversibly at high rates. Moreover, the cytotoxicity of MMS in checkpoint mutants occurs specifically when cells are allowed to enter $S$ phase with DNA damage. Thus, preventing damage-induced DNA replication fork catastrophe seems to be a primary mechanism by which checkpoints preserve viability in the face of DNA alkylation.

MMS slows S phase progression in checkpoint-proficient yeast strains. This might be attributed entirely to the inhibition of late origin firing in these cells ${ }^{5,6}$. Alternatively, a reduced rate of replication fork progression ('fork rate') may also contribute to the slow $\mathrm{S}$ phase. Likewise, the acceleration through $S$ phase in MMS seen in checkpoint mutants may solely be a consequence of their inability to inhibit late origin firing or may be an indication that checkpoints also regulate fork rates. To follow replication forks, we used a density transfer approach (refs 8 and 9 and Supplementary Information). Cells were grown in 'heavy' isotopes $\left({ }^{13} \mathrm{C}\right.$ glucose, ${ }^{15} \mathrm{~N}$ ammonium sulphate) to ensure full isotope substitution of the parental DNA. These cells were arrested in G1 phase with $\alpha$-factor 